\title{
Test-Retest Reliability of Lower Extremity Functional Tests After a Closed Kinetic Chain Isokinetic Testing Bout
}

\author{
Robert C. Manske, Barbara Smith, and Frank Wyatt
}

Context: Athletes are often examined for return to sports using a functional-testing algorithm. No research has determined whether a closed kinetic chain (CKC) isokinetic testing bout influences the reliability of functional tests. Objective: To determine whether a concentric CKC isokinetic test bout alters test-retest reliability of lower extremity functional testing. Design: Subjects participated in velocity-spectrum CKC isokinetic bouts on 2 days 1 week apart. Setting: Hospital-based clinic. Participants: 28 normal subjects (mean age 26.7 years, height $27.2 \mathrm{~cm}$, weight $75.8 \mathrm{~kg}$ ). Analysis and Results: Means and SDs were determined. Intraclass correlation coefficients were used to calculate test-retest reliability and between days 1 and 2 ranged from .91 to 98. Conclusions: Even after a CKC isokinetic test bout, test-retest reliability of functional tests is very high. Future research should determine test-retest reliability of functional tests for patients with lower extremity pathology. Key Words: muscle strength, functional outcome measures, physical-performance tests

Manske RC, Smith B, Wyatt F. Test-retest reliability of lower extremity functional tests after a closed kinetic chain isokinetic testing bout. / Sport Rehabil. 2003;12:119-132. 02003 Human Kinetics Publishers, Inc.

Athletic trainers and sports physical therapists often treat patients after lower extremity injury or surgical intervention. The ultimate goal of rehabilitation is to return these individuals to full premorbid function in the most efficient manner. Clinicians should assess level of impairment with goniometric measurement of range of motion, manual muscle testing, ligament-laxity testing, girth measurements, and isokinetic testing. Although these measurements are crucial to determine the extent of remaining pathology, they do not always provide an indication of a patient's true physical-functional capabilities.

Various functional-performance tests have been developed in an attempt to more accurately quantify physical function. When attempting to quantify

Manske and Smith are with the Dept of Physical Therapy, Wichita State University, Wichita, KS 67260-0043. Wyatt is with the Dept of Health, Human Performance, and Recreation, Baylor University, Waco, TX 76798. 
lower limb impairment, variables such as pain, swelling, crepitus, neuromuscular coordination, muscle strength, muscle endurance, and joint stability play important roles. ${ }^{1}$ Functional testing requires that the patient perform activities that closely simulate a given sport or recreational activity. In addition to a variety of stresses across the lower limb, functional testing assesses whether the patient is mentally ready to return to a given activity. Functional-testing methods should be fast and easy to perform in any setting, require minimal staff or training, ${ }^{2}$ and be sport-specific in nature.

A functional test's reliability is essential to ensure accuracy and repeatability. Reliability is the dependability, reproducibility, and repeatability of a given measurement instrument. ${ }^{3}$ Potential problems can be managed by implementing a standardized protocol with precise instructions and clear operational definitions.

Davies $^{4-5}$ developed a functional-testing algorithm (FTA) as a method to assess a patient in a systematic manner and to establish the functional progression of exercise and testing during the rehabilitative process. In the FTA a sequence of tests must be passed before the patient can progress safely to the next level. Basic tests and measurements form the FTA's first stage. These include but are not limited to palpation, range-of-motion testing, manual muscle testing, flexibility testing, special tests, kinesthesia/ proprioception tests, and KT- 1000 testing. If results of these measurements demonstrate less than a $10 \%$ difference between the involved and uninvolved extremities, the patient is progressed to the next level of testing. The second FTA level includes both closed and open kinetic chain isokinetic testing. If results of these measurements on the involved extremity are within $10 \%$ of those on the uninvolved extremity, the patient is progressed to the next level of testing. It is at this point that the patient undergoes various functional tests. If an individual has not fully passed the FTA, he or she will return to rehabilitation and concentrate on areas in which he or she faltered during the test. For example, if an individual has passed the first phase of basic tests and measures and the isokinetic test yet fails the single-leg hop functional tests, rehabilitation would emphasize single-leg power-type exercises. After appropriate rehabilitation, the athlete will then again be progressed through the entire FTA to ensure that he or she is at least at the same level as during previous testing.

Numerous authors have measured test-retest reliability of functional tests with normal subjects. ${ }^{9-15}$ In all of those studies, the subjects performed a brief warm-up followed by the given functional tests. Theoretically this could be problematic. In most clinical situations an isokinetic test or tests are generally performed before functional testing. Using the FTA for patient progression, patients always undergo isokinetic testing before performing one or more functional tests. The purpose of this study is to determine whether a set of isokinetic testing bouts before functional testing alters test-retest reliability of the functional tests for the lower extremity. 


\section{Methods}

\section{Subjects}

A sample of 28 healthy participants (mean age $26.7 \pm 5.2$ years, range 19 37 ; mean height $27.2 \pm 1.6 \mathrm{~cm}$, range $24.4-29.9$; mean weight $75.8 \pm 14.9 \mathrm{~kg}$, range 47.7-107.1) was recruited through a university physical therapy educational program and a local sports and orthopedic physical therapy outpatient clinic. Participants were evaluated for lower extremity pathology through a self-reported health-history questionnaire and a physical examination by the principal investigator to assess for knee instability via ligamentous testing, and proprioceptive ability was tested with single-legstance testing. Participants had to be able to hold a single-leg stance for 30 seconds without loss of balance to be included in the study. Any participant with acute knee or ankle injury (within the preceding 9 months), past surgical procedure(s) on one or both knees, ligamentous instability, or loss of proprioceptive ability found on exam was excluded from the study. Participants received an explanation of risks, benefits, and procedures and signed an informed consent approved by the university's and medical center's institutional review boards.

\section{Procedures}

A test-retest design was used, with 7 days between testing sessions. Participants were asked to refrain from increasing or substantially changing their present exercise routine to control for outside factors that could contaminate the results. Time of day for testing was kept consistent. In addition, the participants were asked to keep personal habits (eg, sleep, diet) consistent between testing periods. Participants wore shorts and, to rule out footwear as a cause of any difference in scores, they were asked to wear the same athletic shoes for all tests. The functional-testing surface was all low-pile carpet glued onto a concrete floor. The functional tests used were chosen based on their prevalence in the orthopedic and sportsmedicine literature. Leg dominance was determined by asking participants which leg they would be most comfortable kicking a ball with. All testing was performed in a nonrandomized order, beginning with the isokinetic bouts, followed by the functional tests. This was done to more closely simulate actual clinical conditions. Three sports and orthopedic physical therapists, each with a minimum of 6 years of clinical experience, performed all data collection.

\section{Instrumentation}

The Lido Linea closed kinetic chain isokinetic dynamometer (Loredan Biomechanical, West Sacramento, Calif) was used to test the leg extensors before functional testing. The Lido Linea was calibrated per the instruction manual. Calibration procedures have heen described previously. ${ }^{16}$ 


\section{Warm-Up}

Each participant performed a warm-up before each session, consisting of 5 minutes of exercise on a stationary bicycle at a self-selected, submaximal intensity.

\section{Isokinetic Testing}

Each participant was positioned semirecumbent in the Linea's leg-press chair and stabilized by a lap belt (Figure 1). With the knees fully extended, the participant's hips were in approximately $70^{\circ}$ of flexion. The linear range of motion was set so that the knees moved from approximately $90^{\circ}$ to $5^{\circ}$ of knee flexion during the complete leg-press stroke. A popliteal pad was placed behind the knees to prevent hyperextension. The range-of-motion stops and the popliteal pad's position were recorded at the first session and replicated during the second test session. Participants were allowed to grasp the system's handles during testing.

Before each isokinetic bout, the participant performed an isokinetic warm-up of 3 gradient, submaximal repetitions, plus 1 maximal repetition at each test velocity. Each participant performed the same isokinetic bouts,

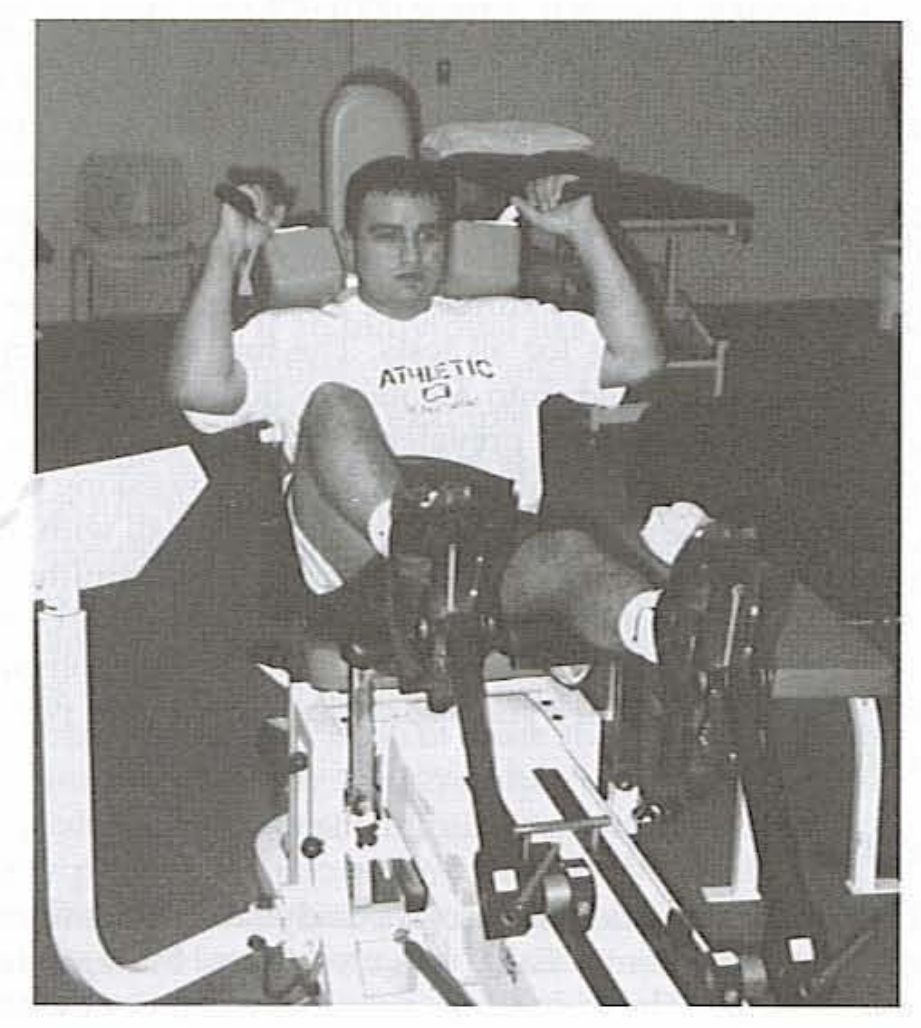

Figure 1 Semirecumbent position in Lido Linea. 
which consisted of 3 bilateral coupled leg-press sets followed by 3 bilateral reciprocal leg-press sets. A bilateral coupled leg press on the Lido Linea is defined as a complete leg-press stroke from the flexed to the extended position with both extremities in unison. A bilateral reciprocal leg press is defined as a complete leg-press stroke from the flexed-knee to the extended position for each limb in direct succession. Each isokinetic bout consisted of 6 repetitions at 3 different velocities. A velocity spectrum of isokinetic leg presses was performed in the concentric mode, consisting of slow (25.4 $\mathrm{cm} / \mathrm{s})$, medium $(50.8 \mathrm{~cm} / \mathrm{s})$, and fast $(76.2 \mathrm{~cm} / \mathrm{s})$ velocities. Participants were instructed to perform 6 successive maximal volitional concentric isokinetic repetitions at each velocity. Strong verbal encouragement was provided in a consistent manner to elicit maximal effort. Participants were also given visual feedback of their performance during the exercise session. The exercise bout progressed from slow to medium to fast velocity, which is common in clinical isokinetic testing. Efforts were separated by 30 -second rest periods.

At the conclusion of the isokinetic bouts, each participant performed light quadriceps and hamstring stretching for up to 3 minutes. The cooldown was followed by the functional-testing sequence. A brief cool-down was performed immediately after the isokinetic test, again to more closely simulate clinical conditions. During the actual FTA it generally takes several minutes to print out results of the isokinetic test to determine whether an individual has adequate strength to continue through the testing procedures.

\section{Functional Testing}

After the closed kinetic chain isokinetic bouts, each participant performed the following functional tests: 2-legged vertical jump, single-leg vertical jump, 2-legged jump for distance, single-leg hop, and timed 6-m single-leg hop. Functional tests were all performed in the same sequence. Again, this sequence was used to more closely replicate the FTA described previously. The functional tests are progressed sequentially with increasing complexity. Three warm-up trials were allowed for each functional test to enable the participants to gain some familiarity with the actions required and to ensure competence and understanding of test instructions. Each participant performed 3 attempts of each test and was asked to give maximal effort for each attempt. A 1-minute rest period was allowed between tests, and up to 30 seconds rest were given as needed between the 3 jumps. Upper extremity movement was restricted as much as possible. After completing functional testing, participants performed a cool-down by riding a stationary bicycle and/or stretching.

Vertical Jump. The vertical jump is commonly used in exercise physiology to measure lower extremity power. It is generally accepted that a large amount of error can be generated by using traditional chalk or tape methods of assessing vertical jump, so we used the Vertec jump apparatus (Sports 
Imports, Inc, Columbus, Ohio). A standing baseline-reach measurement was initially performed with each participant standing flatfooted and reaching as high as possible with the right and then the left upper extremity. Then the participant was asked to jump as high as possible and to reach with the ipsilateral upper extremity to displace a movable plastic vane of 0.5 -in increments. Countermovements with the upper extremities were used during this test because reaching is part of the testing procedure to obtain a measurement. The standing baseline reach was subtracted from the total vertical-jump score to obtain the distance jumped. Measurements were made bilaterally and unilaterally.

Jump and Hop Tests. The 2-legged-jump test and the single-leg-hop test were performed in a similar manner. A standing long-jump mat (SBP, Toronto, Canada) composed of a durable rubber material was used for measuring jumps and hops. This mat has a standardized starting position and enables measurement of jumps of $50-320 \mathrm{~cm}$. Participants stood with

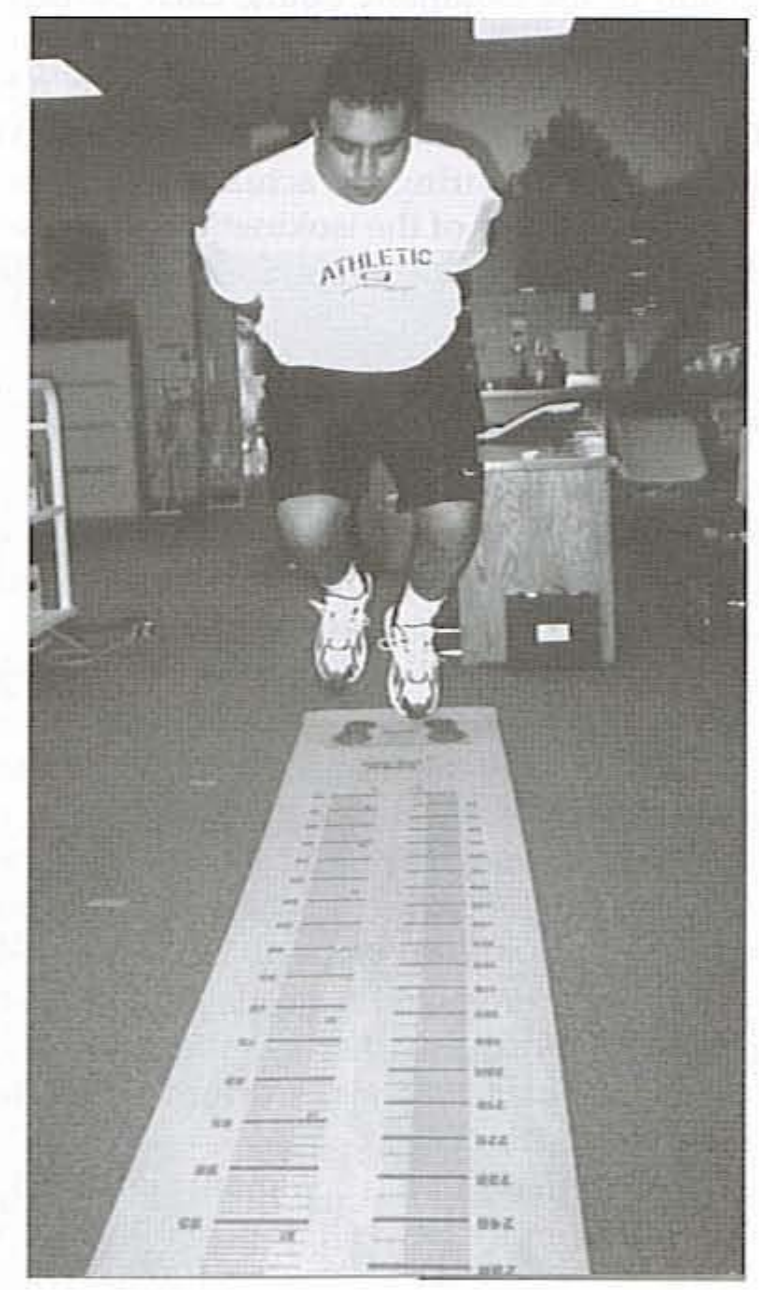

Figure 2 Double-leg jump for distance. 


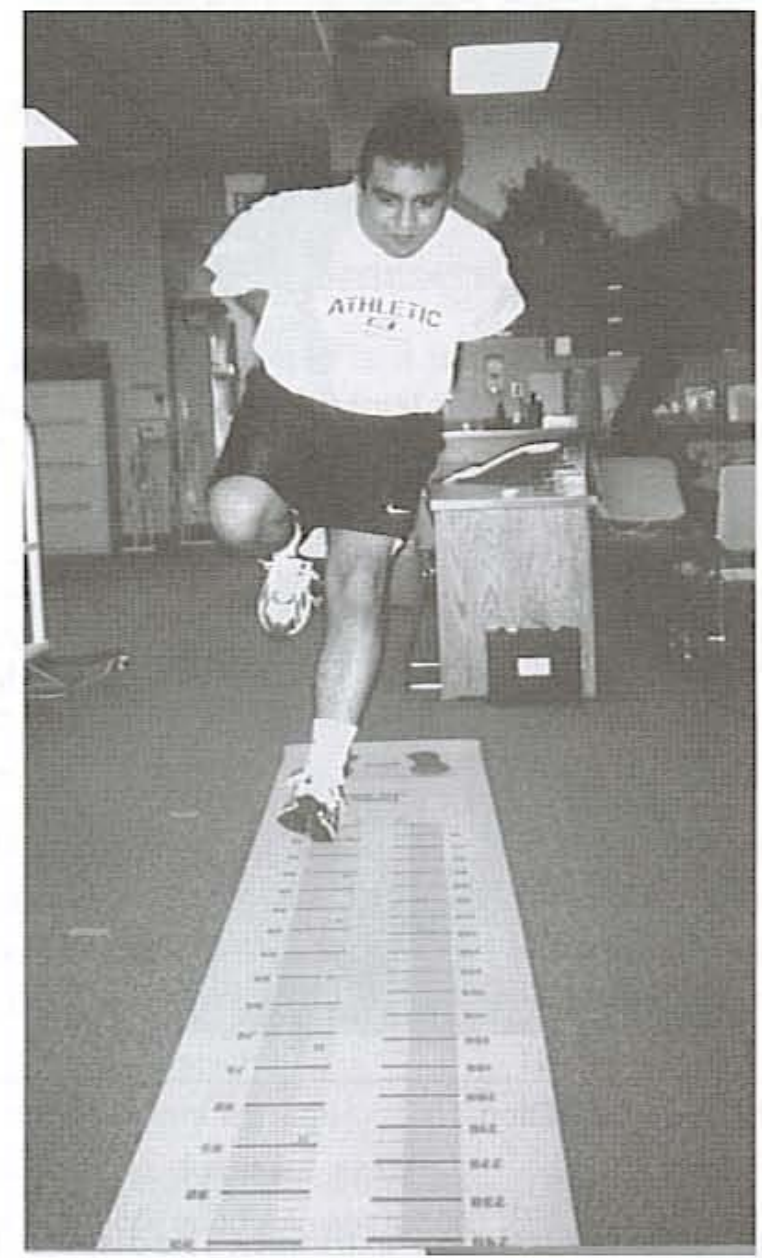

Figure 3 Single-leg hop for distance.

the front of their shoes behind the starting mark with hands on hips and knees bent. They then jumped or hopped (2-legged or single-leg; Figures 2 and 3) as far horizontally as possible, landing with bent knee(s) to help decrease the risk of knee injury. The spot where the heel of the more posterior shoe landed was marked and used as the jump distance. To qualify for a legitimate jump, a participant had to land without falling down or taking a step. After landing, this position had to be held for at least 2 seconds. A failed jump included loss of balance, touching the floor with the contralateral upper or lower extremity (during single-leg hop), or an additional short hop after landing. The mean distance of 3 maximal attempts of each test was recorded.

During the single-leg hop, participants hopped using the nondominant leg first, then the dominant leg. Once again, as with the 2-legged jump test, they held their hands on their hips to avoid gaining momentum with the arms. They then bent their knees and hopped as far forward as possible, 


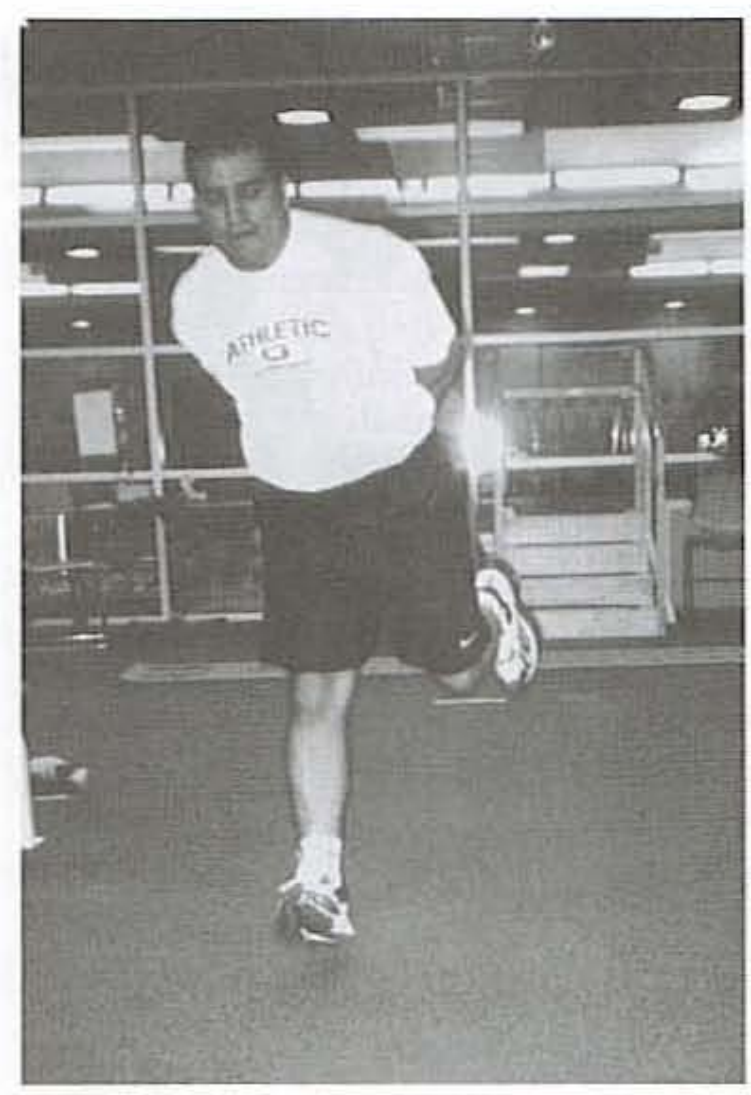

Figure 4 Single-leg 6-m timed hop.

this time landing on the contralateral extremity. The distance from the starting mark to the heel of the landing leg was measured. Each participant had to land without losing balance and without relying on the other foot or upper extremity to aid in landing.

Single-Leg 6-m Timed Hop. The single-leg 6-m timed hop began at the same spot as the single- and 2-legged jump/hop tests. Participants used large, forceful single-leg hopping motions across the 6-m distance (Figure 4). A standard manual stopwatch was used to time each trial to the nearest 0.01 second. Three trials were performed with each lower extremity, with the nondominant leg tested first, then the dominant leg.

\section{Data Analysis}

Statistical analysis was performed using Statistical Package for Social Sciences (SPSS, version 9.0, Chicago, Ill). Analysis included standard descriptive information (eg, mean, SD), as well as correlation coefficients for each functional test. The means of day 1 and day 2 test scores were used to determine intraclass correlation coefficients $\left(\mathrm{ICC}_{2,1}\right)$. The alpha level for all tests was set at $\leq .05$. 


\section{Results}

Descriptive statistics for each functional test are shown in Table 1. The ICCs for the functional tests ranged from .91 to .98 (Table 2).

\section{Comments}

Reliability of any functional test is a prerequisite to its use in a clinical setting. To date no study has examined the effect that a closed kinetic chain isokinetic test might have on the test-retest reliability of functional tests.

\section{Table 1 Means, Standard Deviations, and Ranges} of Functional-Test Scores*

\begin{tabular}{lrrc}
\hline & Mean & SD & Range \\
\hline Vertical jump, bilateral (cm) & 42.7 & 10.9 & $27.2-64.8$ \\
Vertical jump, dominant leg (cm) & 27.4 & 8.4 & $17.0-44.5$ \\
Vertical jump, nondominant leg (cm) & 31.5 & 10.4 & $17.8-57.7$ \\
2-legged jump, bilateral (cm) & 162.1 & 26.7 & $110.5-224.0$ \\
Single-leg hop, dominant leg (cm) & 126.5 & 21.6 & $88.1-185.4$ \\
Single-leg hop, nondominant leg (cm) & 127.8 & 28.2 & $86.4-188.0$ \\
Single-leg timed 6-m hop, dominant leg (s) & 2.1 & 0.36 & $1.7-2.9$ \\
Single-leg timed 6-m hop, nondominant leg (s) & 2.1 & 0.42 & $1.49-3.33$ \\
\hline
\end{tabular}

*B indicates bilateral; DL, dominant leg; and NDL, nondominant leg.

\section{Table 2 Functional-Test Test-Retest Reliability}

\begin{tabular}{lc}
\hline Functional test & ICC \\
\hline Vertical jump, bilateral & .98 \\
Vertical jump, unilateral, dominant leg & .98 \\
Vertical jump, unilateral, nondominant leg & .98 \\
2-legged hop for distance, dominant leg & .91 \\
Single-leg hop for distance, dominant leg & .96 \\
Single-leg hop for distance, nondominant leg & .97 \\
Single-leg timed hop, dominant leg & .92 \\
Single-leg timed hop, nondominant leg & .96
\end{tabular}


The purpose of this study was to determine whether a closed kinetic chain isokinetic test would alter the test-retest reliability of several commonly used functional-testing procedures.

This study's test-retest reliability is consistent with those of others that did not use an isokinetic bout before implementing functional tests in a similar order as indicated in the FTA. Because of facility constraints, the entire FTA as described by Davies ${ }^{4-8}$ was not used. In addition, the singleleg 6-m timed hop was used instead of the lower extremity functional test. From this study and the results of other studies (Table 3 ) it appears that reliability of these functional tests is not affected by lower extremity closed kinetic chain testing in normal subjects.

Although most of the test-retest reliability scores of the studies in Table 3 are high, numerous variables (eg, length of rest periods, number of trials performed) are different. Therefore, it is unlikely that generalizations across functional tests can be made. Hypothetically this should be expected and acknowledged. In the real-word everyday clinic, variations exist. Not all clinics have the same equipment. Some might use chalk markings to measure vertical jump, and others might use the Vertec apparatus, as in the present study. Some might have a jump mat, and others use carpet or cement flooring. Despite this variability of testing methods, it can be seen from Table 3 that the single-leg hop is the most-studied form of functional test. This could be because researchers and clinicians can more easily compare the dominant with nondominant or injured with uninjured extremity. This is also the case with the single-leg timed 6-m hop, although reliability is highly variable with this test. Potential variations in reliability might result from differences in inclusion of and number of practice sessions among the studies.

The current study revealed test-retest ICCs ranging from .91 to .98 for the group of functional tests used. This demonstrates excellent reliability of the functional tests measured. ICCs in the .90-1.0 range are considered excellent in the field of rehabilitation. ${ }^{17.18}$

In the present study, reliability scores were lower for the dominant leg than for the nondominant leg. This could be because the dominant leg is usually used in an open-chain fashion, whereas the nondominant leg is often used in a stable weight-bearing fashion during higher-level activities. A difference between the present study and that of Bolga and Keskula ${ }^{10}$ is that testing order was randomized in the latter but not in the current study. When using the FTA, one progresses from one functional test to the next if criteria are met. The testing order is not randomized. Therefore, functional tests were performed in an order that more closely simulated real-life clinical situations. Theoretically this could have allowed subjects an edge because, after the first testing session, they knew which tests and in which order they would be performing on subsequent testing dates.

Establishing test-retest reliability is essential to ensure accurate longitudinal results. The main purpose of this study was to determine whether 


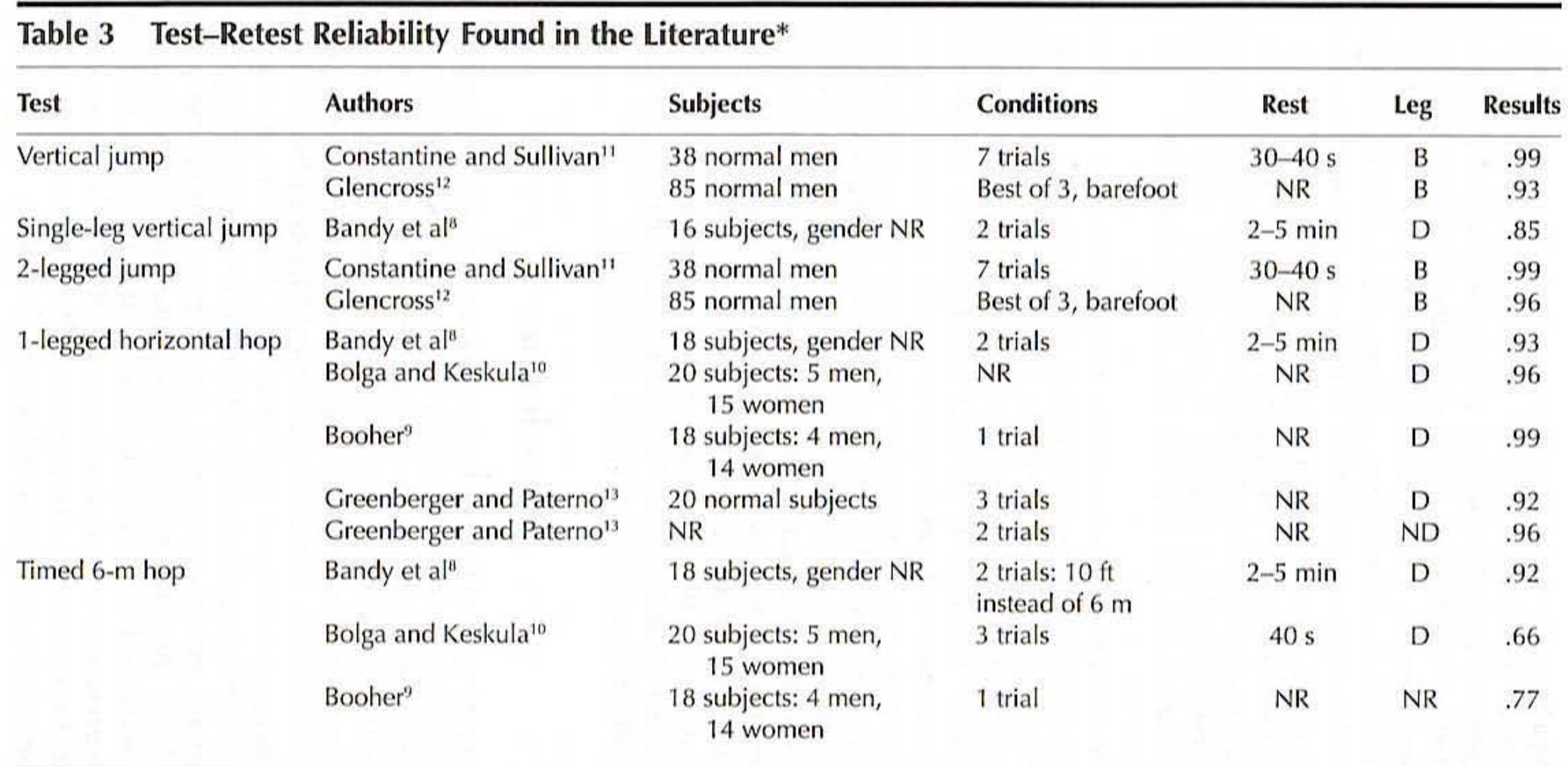

*B indicates bilateral; NR, not reported; D, dominant; and N, nondominant. 
completing a battery of isokinetic tests would effect a change in test-retest reliability of various lower extremity functional tests. The results of the study suggest that an isokinetic bout does not alter reliability of functional tests that follow. Two reasons could explain the findings from this study.

We thought that the isokinetic protocol might have fatigued the lower leg musculature, thereby inhibiting performance on the FTA that followed. After the isokinetic bouts, subjects in all instances demonstrated increased respiration and heart rate and fatigued legs per subjective reports to the primary author. One would assume that after 6 sets of 6 repetitions of full volitional effort on a closed kinetic chain isokinetic dynamometer, subjects' lower extremities would be fatigued. Despite this, it is possible that fatigue was not achieved and therefore did not affect performance on the functional tests.

A second, more plausible, reason that the isokinetic bout might not have altered test-retest reliability is that short yet adequate rest periods were given during each isokinetic bout and during functional testing. During the isokinetic bouts and the functional testing, no single test lasted more than 8 seconds. This type of physical activity stresses mainly the phosphagen energy system, ${ }^{19}$ which works primarily while one is performing short-duration, all-out maximal-effort exercise. There is generally enough energy in the active muscles to perform short-duration, all-out powerful activities such as those performed in this study. ${ }^{19}$ This form of energy use has a very short recovery time, allowing subjects to replenish their energy stores before the next test. Intense exercise bouts that last approximately 10 seconds, followed by a 5-second rest recovery, can be performed for as long as 30 minutes without undue fatigue. ${ }^{20}$ Therefore, as long as an adequate recovery period is given, functional testing using up to 3 or 4 tests after an isokinetic bout or bouts is very appropriate.

\section{Conclusion}

The primary purpose of this study was to determine whether an isokinetic bout would affect test-retest reliability of selected functional tests that follow it. From the results of this study and others that have determined testretest reliability without isokinetic testing, it appears that a closed kinetic chain isokinetic bout before functional testing does not alter reliability of those functional tests. Thus, using a clinical FTA described by Davies ${ }^{9-15}$ will not change reliability of functional tests that follow it. A short rest period between individual test repetitions and between testing bouts allows adequate recovery to combat any fatigue from testing or a prior isokinetic bout. Thus, in normal, healthy, young populations, an FTA is an appropriate method of determining functional outcomes in a clinical setting. Further research is required to determine whether similar results would be obtained in populations receiving rehabilitation for lower extremity injuries commonly seen in sports and orthopedic clinics. 


\section{Acknowledgments}

The authors gratefully thank Mark Needham, Julie Oler-Manske, and Kim Yearout for their assistance during this project.

\section{References}

1. Barber SD, Noyes FR, Mangine RE, McCloskey JW, Hartman W. Quantitative assessment of functional limitations in normal and anterior cruciate ligament deficient knees. Clin Orthop. 1990;255:204-214.

2. Manske RC, Vequist $\mathrm{S}$. Examination of the knee with special and functional testing. In: Wadsworth C, ed. Disorders of the Knee. American Physical Therapy Association, Orthopedic Section Home Study Course. Lawrence, Kan: Allen Press; 2001.

3. Currier DP. Elements of Research in Physical Therapy. 3rd ed. Baltimore, Md: Williams \& Wilkins; 1990.

4. Davies GJ. The need for critical thinking in rehabilitation. J Sport Rehabil. 1995;4:1-22.

5. Davies GJ. Functional testing algorithm for patients with knee injuries [abstract]. In Proceedings of the 12th International Congress of the World Confederation for Physical Therapy. Alexandria, Va: American Physical Therapy Association: $1995 ; 912$.

6. Davies GJ, Malone T. Proprioception, open kinetic chain and closed kinetic chain exercises and their application to rehabilitation and assessment. In: Instructional Course Lectures. Rosemount, Ill: American Orthopedic Society for Sports Medicine; 1992:19.

7. Davies GJ, Wilk KE, Ellenbecker TS. Assessment of strength. In: Malone TR, McPoil T, Nitz AJ, eds. Orthopedic and Sports Physical Therapy. 3rd ed. St Louis, Mo: Mosby Year Book Inc; 1997:225-227.

8. Bandy WD, Rusche KR, Tekulve FY. Reliability and limb symmetry for five unilateral functional tests of the lower extremities. Isokinet Exerc Sci. 1994;4:108111.

9. Booher LD, Hench KM, Worrell TW, Stikeleather J. Reliability of three singleleg hop tests. J Sport Rehabil. 1993;2:165-170.

10. Bolga LA, Keskula DR. Reliability of lower extremity functional performance tests. J Orthop Sports Phys Ther. 1997;3:138-142.

11. Considine WJ, Sullivan WJ. Relationship of selected tests of leg strength and leg power on college men. Res Q. 1973;44:404-416.

12. Glencross DJ. The nature of the vertical jump test and the standing broad jump. Res Q. 1966;37:353-359.

13. Greenberger HB, Paterno MV. The test-retest reliability of a one-legged hop for distance in healthy young adults [abstract]. J Orthop Sports Phys Ther. 1994;1:62.

14. Hu HS, Whitney SI, Irrgang J, Janosky J. Test-retest reliability of the one-legged vertical jump test and the one-legged standing hop test [abstract]. J Orthop Sports Phys Ther. 1992;15:51. 
15. Risberg MA, Holm I, Ekeland A. Reliability of functional knee tests in normal athletes. Scand J Med Sci Sports. 1995;5:24-28.

16. Davies GJ, Heiderscheit B. Validity and reliability of the Lido Linea closed kinetic chain isokinetic dynamometer. J Orthop Sports Phys Ther. 1997;2:133136.

17. Domholt E. Physical Therapy Research: Principles and Applications. 2 nd ed. Philadelphia, Pa: WB Saunders; 2000.

18. Johnson BL, Nelson JK. Practical Measurements for Evaluation in Physical Education. Edina, Minn: Burgess; 1974.

19. McArdle WD, Katch FI, Katch VL. Exercise Physiology. Energy, Nutrition, and Human Performance. 4th ed. Baltimore, Md: Williams \& Wilkins; 1996.

20. Christensen EH, Hedman R, Saltin B. Intermittent and continuous running. Acta Physiol Scand. 1960;50:269. 
